\title{
Erratum
}

Frédéric Bihan · Matthias Franz · Clint McCrory · Joost van Hamel

\section{Is every toric variety an M-variety?}

manuscripta math. (2006)

Digital Object Identifier (DOI) 10.1007/s00229-006-0004-z

Published online: 17 June 2006

Due to a processing error the author's name was spelled incorrectly. The correct name should be Joost van Hamel. The error was only in the online version.

The online version of the original article can be found at http://dx.doi.org/10.1007/s00229006-0004-z

F. Bihan: Laboratoire de Mathématiques, Université de Savoie, 73376 Le Bourget-du-Lac Cedex, France

M. Franz: Fachbereich Mathematik, Universität Konstanz, 78457 Konstanz, Germany

C. McCrory: Mathematics Department, University of Georgia, Athens GA 30602, USA

J. van Hamel (凶): Departement Wiskunde, K.U. Leuven, Celestijnenlaan 200B, 3001 Leuven (Heverlee), Belgium. e-mail: Joost.vanHamel@wis.kuleuven.be 